\title{
Short cycle covers of graphs with at most $77 \%$ vertices of degree two
}

\author{
Anna Kompišová* Robert Lukotka ${ }^{\dagger}$ \\ Faculty of Mathematics, Physics and Informatics \\ Comenius University \\ Bratislava, Slovakia \\ \{kompisova, lukotka\}@dcs.fmph.uniba.sk
}

Submitted: Jan 13, 2020; Accepted: Oct 18, 2020; Published: Nov 13, 2020

(C) The authors. Released under the CC BY license (International 4.0).

\begin{abstract}
Let $G$ be a bridgeless multigraph with $m$ edges and $n_{2}$ vertices of degree two and let $c c(G)$ be the length of its shortest cycle cover. It is known that if $c c(G)<1.4 m$ in bridgeless graphs with $n_{2} \leqslant m / 10$, then the Cycle Double Cover Conjecture holds. Fan (2017) proved that if $n_{2}=0$, then $c c(G)<1.6258 m$ and $c c(G)<1.6148 m$ provided that $G$ is loopless; morever, if $n_{2} \leqslant m / 30$, then $c c(G)<1.6467 m$. We show that for a bridgeless multigraph with $m$ edges and $n_{2}$ vertices of degree two, $c c(G)<1.6148 m+0.0741 n_{2}$. Therefore, if $n_{2}=0$, then $c c(G)<1.6148 m$ even if $G$ has loops; if $n_{2} \leqslant m / 30$, then $c c(G)<1.6173 m$; and if $n_{2} \leqslant m / 10$, then $c c(G)<$ $1.6223|E(G)|$. Our improvement is obtained by randomizing Fan's construction.
\end{abstract}

Mathematics Subject Classifications: 05C38, 05C70

\section{Introduction}

A cycle is a graph with all vertices of even degree and a circuit is an inclusion-wise minimal nonempty cycle. The length of a cycle/circuit $C$ is the number of its edges. A collection of cycles of a graph $G$ covers $G$ if each edge of $G$ is contained in at least one of the cycles. Such a collection is called a cycle cover. The length of a cycle cover is the sum of lengths of its cycles. By $c c(G)$ we denote the length of the shortest cycle cover of $G$. Note that a graph has a cycle cover if and only if it is bridgeless.

Graphs in this paper may have loops and parallel edges. Let $G$ be a bridgeless graph with $m$ edges and $n_{2}$ vertices of degree 2 . The best known general upper bound,

* Supported by VEGA 1/0813/18.

†Supported by APVV-19-0308, and by VEGA 1/0813/18. 
$c c(G) \leqslant 5 m / 3$, was independently established by Alon and Tarsi [1] and Bermond, Jackson and Jaeger [2] more than 35 years ago. On the other hand the Shortest Cycle Cover Conjecture $[1,11]$ asserts that $c c(G) \leqslant 1.4 m$ and is tight for infinitely many graphs. This conjecture is surprisingly strong as it implies the well-known Cycle Double Cover Conjecture [9]. This remains true even if we restrict the Shortest Cycle Cover Conjecture to subcubic graphs with at most $m / 10$ vertices of degree 2 .

Due to the lack of progress in the general case, much attention has been paid to special classes of graphs. Among the most general classes considered are bridgeless graphs with minimal degree 3: Kaiser et al. [10] proved that $c c(G)<1.6296 m$ for loopless graphs which in turn Fan [6] improved to $c c(G)<1.6148 m$ in the loopless case and to $c c(G)<1.6257 m$ if $G$ is allowed to have loops. Results for graphs with loops can be easily transformed to results for graphs where a small number of vertices of degree 2 is allowed - we may just add loops to the vertices of degree 2 . In this way Fan [6] proved that if $n_{2} \leqslant m / 30$, then $c c(G)<1.6467 m$. However, if we only know that $n_{2} \leqslant m / 10$, then the general upper bound beats the bound obtained by Fan.

We improve Fan's result by proving following theorem.

Theorem 1. Let $G$ be a bridgeless graph (loops allowed) with $m$ edges and $n_{2}$ vertices of degree 2. Then $c c(G)<1.6148 m+0.0741 n_{2}$.

Therefore, if $n_{2}=0$, then $c c(G)<1.6148 m$ even if $G$ has loops; if $n_{2} \leqslant m / 30$, then $c c(G)<1.6173 m$; and if $n_{2} \leqslant m / 10$, then $c c(G)<1.6223 m$. Theorem 1 gives better results than the general bound whenever $n_{2} \leqslant 0.7000 \mathrm{~m}$, which is always the case when $n_{2} \leqslant 0.7777|V(G)|$.

The proof of Theorem 1 essentially follows Fan's proof. However, on several places where a choice is made, instead of an arbitrary selection we make a random one (from a defined distribution). Then we analyze the expected length of the resulting cycle cover. As many parts of the proof are identical with Fan's proof, we keep the proofs short. We refer the reader to [6] for further details.

\section{The proof}

We prove the following theorem which implies Theorem 1 (it suffices to add one loop to each vertex of degree two). The degree of a vertex equals the number of incident non-loop edges plus twice the number of incident loops.

Theorem 2. Let $G$ be a bridgeless graph of minimum degree 3 which contains $m$ non-loop edges and $s$ loops. Then $c c(G)<218 / 135 \cdot m+29 / 27 \cdot s$.

A graph is called 5-odd-edge-connected if it includes no odd edge-cuts of order smaller than 5. A proper spanning cycle of a graph $G$ is a spanning cycle $F$ of $G$ such that each component has an edge, each component with a loop has only one edge (the loop), and $G / F$ is 5-odd-edge-connected. 
Lemma 3. Let $G$ be a bridgeless graph on at least two vertices with minimum degree 3 such that every loop is incident with a vertex of degree 4 . There exists a probability distribution over proper spanning cycles $F$ of $G$ such that for every edge e we have $P(e \in F)=2 / 3$ if it is a non-loop and $P(e \in F)=1 / 3$ if it is a loop.

Proof. For each vertex $v$ of $G$ which is not of degree 3 we sequentially replace $v$ without affecting other incidences as follows. Let us denote $G_{s}$ the graph before the operation and $G_{e}$ the graph after the operation. If $v$ is incident with a loop (and thus $v$ is incident with exactly two other edges), we replace $v$ and the loop with a 2-circuit, so that both vertices of the 2-circuit have degree 3 (Figure 1a). Thus if we contract an edge of the new 2-circuit in $G_{e}$ into a single vertex $v$, then we get $G_{s}$. Otherwise, we replace $v$ by a tree in such a way that the new vertices have degree 3 and $G_{e}$ is bridgeless (Figure 1b). Thus if we contract the tree in $G_{e}$ into a vertex $v$ we obtain $G_{s}$. We can always perform this operation without introducing a bridge due to the Splitting Lemma (Lemma III.26 in [7]). After performing this operation for all vertices we get a bridgeless cubic graph. We denote it $G_{c}$.

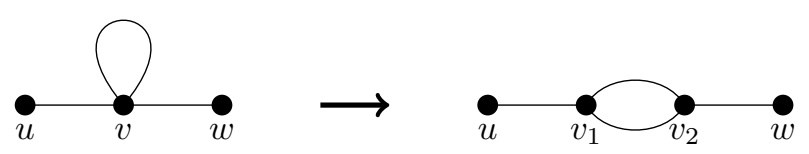

(a)
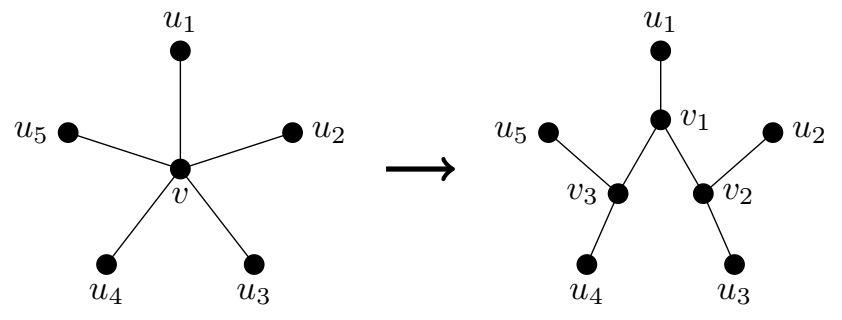

(b)

Figure 1: Transformation of vertices which are not of degree 3.

The characterization of the perfect matching polytope by Edmonds [5] implies (see Lemma 10 in [4]) that for each bridgeless cubic graph there exists a probability distribution over the set of its 2-factors of such that each edge is present in a random 2-factor with probability $2 / 3$. Let $\mathcal{D}_{c}$ be such distribution for $G_{c}$. Consider a 2 -factor $F_{c}$ which appears in $\mathcal{D}_{c}$ with non-zero probability. Note that $G_{c} / F_{c}$ is 5-odd-edge-connected. Indeed, let $X$ be a 3 -cut of $G_{c}$. By parity, $\left|X \cap F_{c}\right| \in\{0,2\}$. As each edge is present in $F_{c}$ with probability $2 / 3$, the linearity of expectation implies that $\mathbb{E}\left(\left|X \cap F_{c}\right|\right)=2$, and therefore $P\left(\left|X \cap F_{c}\right|=0\right)=0$.

Pick at random a 2-factor $F_{c}$ of $G_{c}$ from $\mathcal{D}_{c}$. Let us contract all trees which we have introduced in the beginning the proof. For each introduced 2-cycle we contract one of its edges. If one of the edges is in $F_{c}$ and the other is not, we contract the edge from $F_{c}$. 
In this way we, again, obtain $G$ and denote the subgraph obtained from $F_{c}$ by $F$. The subgraph $F$ obtained by this procedure defines a probability distribution $\mathcal{D}$ of subgraphs of $G$. We show that this distribution satisfies the lemma statement.

As $G_{c} / F_{c}$ is 5 -odd-edge-connected, so is $G / F$. Similarly, as we contract a forest, a spanning cycle where each component has an edge remains a spanning cycle where each component has an edge. Finally, due to the choice made while contracting the introduced 2-cycles all loops of $F$ are isolated components. Thus $F$ is a proper spanning cycle.

Each non-loop edge appears in $F$ with exactly the same probability as it appears in $F_{c}$, which is 2/3. A loop incident with a vertex $v$ appears in $F$ if and only if a non-loop edge incident with $v$ (chosen arbitrarily) does not appear in $F$ (and thus neither in $F_{c}$ ). This occurs with probability $1 / 3$.

A $\mathbb{Z}_{2} \times \mathbb{Z}_{2}$-flow on a graph $G$ is a function $f: E(G) \rightarrow \mathbb{Z}_{2} \times \mathbb{Z}_{2}$ such that for every vertex the sum of the values of its incident edges is 0 . Compared to the standard definition of flow, $\mathbb{Z}_{2} \times \mathbb{Z}_{2}$ contains only involutions, thus we omit the orientation. For convenience, we denote the elements of $\mathbb{Z}_{2} \times \mathbb{Z}_{2}$ as $\mathrm{R}, \mathrm{G}, \mathrm{B}$, and 0 . We define $f^{-1}(X, H)=\{e \in H \mid f(e) \in X\}$, where $X \subseteq \mathbb{Z}_{2} \times \mathbb{Z}_{2}$ and $H$ is either a subgraph or a set of edges of $G$. A flow $f$ on $G$ is called nowhere-zero if $f^{-1}(\{0\}, G)=\emptyset$.

Consider a bridgeless graph of minimal degree 3. Fix a proper spanning cycle $F$ of $G$. Let $d_{i}$ be the number of components of $F$ of size $i$. For simplicity, in the rest of the text, by $|H|$, where $H$ is a graph, we mean $|E(H)|$, and in $X-Y$ and $X \cap Y$, if $X$ and/or $Y$ is a graph, then we take $E(X)$ and/or $E(Y)$ as the operand in the operation.

As $G / F$ is 5-odd-edge-connected, there exists a nowhere-zero $\left(\mathbb{Z}_{2} \times \mathbb{Z}_{2}\right)$-flow on $G / F$ [8]. We use the following Fan's lemma to modify the flow.

Lemma 4. [6, Lemma 2.2, Theorem 3.3] Let $G$ be a graph, let $f$ be a $\left(\mathbb{Z}_{2} \times \mathbb{Z}_{2}\right)$-flow on $G$ and let $C$ be a cycle of $G$. Then there exists a flow $g$ such that

- $\left|f^{-1}(0, G-C)\right|=\left|g^{-1}(0, G-C)\right|$

- $\left|g^{-1}(0, C)\right| \leqslant|C| / 4$ and if $|C|<20$, then this inequality is strict.

By sequentialy applying this lemma to each component of $F$ we get a flow $f_{0}$ such that for each component $C$ of $F, f_{0}^{-1}(\{0\}, C) \leqslant|C| / 4$ and if $|C|<20$, then this inequality is strict. We permute the elements $\mathrm{R}, \mathrm{G}, \mathrm{B}$ in $f_{0}$ using a random permutation (picked uniformly from the six choices) and call the resulting flow $f$. Let $S$ be the set of loops of $G$.

Claim 5. The graph $G$ has a cycle cover of length at most

$$
2|G-F-S|+|S-F|+|F|+\sum_{i=2}^{\infty} 2\left\lfloor\frac{i}{4}\right\rfloor d_{i}-\sum_{i \in\{4,8,12,16\}} 2 d_{i} .
$$

Proof. We define a cycle cover based on $f$ consisting of three cycles $C_{1}, C_{2}$, and $C_{3}$ as follows:

$$
E\left(C_{1}\right)=f^{-1}(\{\mathrm{R}, \underline{\mathrm{G}}\}, G-F-S) \cup f^{-1}(\{0, \mathrm{~B}\}, F) \cup(S-F),
$$




$$
\begin{aligned}
& E\left(C_{2}\right)=f^{-1}(\{\mathrm{R}, \mathrm{B}\}, G-F-S) \cup f^{-1}(\{0, \underline{\mathrm{G}}\}, F), \\
& E\left(C_{3}\right)=f^{-1}(\{\underline{\mathrm{G}}, \mathrm{B}\}, G-F-S) \cup f^{-1}(\{0, \mathrm{R}\}, F) ;
\end{aligned}
$$

Given a $\left(\mathbb{Z}_{2} \times \mathbb{Z}_{2}\right)$-flow two distinct nonzero elements induce a cycle. Note that $C_{1}\left(C_{2}\right.$, $\left.C_{3}\right)$ is a symetric difference of two cycles: $f^{-1}(\{\mathrm{R}, \underline{\mathrm{G}}\}, G-(S-F))$ and $F \cup(S-F)$ $\left(f^{-1}(\{\mathrm{R}, \mathrm{B}\}, G-(S-F))\right.$ and $F, f^{-1}(\{\underline{G}, \mathrm{~B}\}, G-(S-F))$ and $\left.F\right)$ and thus $C_{1}\left(C_{2}, C_{3}\right)$ is, indeed, a cycle. The total length of the cover is $2 \cdot|G-F-S|+|F|+2 \cdot\left|f^{-1}(\{0\}, G)\right|+|S-F|$. The claim follows from the bound on $\left|f^{-1}(\{0\}, C)\right|$ for each component $C$ of $F$.

Claim 6. The graph $G$ has a cycle cover of length at most

$$
|G-F-S|+|S-F|+|F|+d_{1}+\sum_{i=2}^{\infty}\left(\left\lfloor\frac{i}{2}\right\rfloor+3\right) d_{i}
$$

Proof. We restrict $f$ to $G / F$. The restricted flow is nowhere-zero. As long as there is a cycle consisting of edges with flow value $\mathrm{R}$ or a cycle consisting of edges with flow value $\mathrm{G}$, we change the flow values on all the edges in the cycle to $\mathrm{B}$; this is still a flow on $G / F$. After this procedure, both edges with flow value $\mathrm{R}$ and edges with flow value $\mathrm{G}$ will constitute a forest in $G / F$. We extend the resulting flow to a flow $g$ on $G$ arbitrarily.

Note that, if we add an arbitrary value from $\mathbb{Z}_{2} \times \mathbb{Z}_{2}$ to the flow values of all edges in a component of $F$, the resulting function is still a flow. Thus we can pick $g$ in such a way that $\left|g^{-1}(\{\mathrm{R}, \mathrm{B}\}, C)\right| \leqslant|C| / 2$ for each component $C$ of $F$ and thus

$$
\left|g^{-1}(\{\mathrm{R}, \mathrm{B}\}, F)\right| \leqslant \sum_{i=1}^{\infty}\lfloor i / 2\rfloor d_{i}
$$

We define a cycle cover based on $g$ consisting of three cycles $C_{1}, C_{2}$, and $C_{3}$ as follows:

$$
\begin{aligned}
& E\left(C_{1}\right)=g^{-1}(\{\mathrm{R}, \mathrm{G}\}, G-F-S) \cup g^{-1}(\{\mathrm{R}, \mathrm{G}\}, F) \cup(S-F), \\
& E\left(C_{2}\right)=g^{-1}(\{\mathrm{R}, \mathrm{G}\}, G-F-S) \cup g^{-1}(\{0, \mathrm{~B}\}, F), \\
& E\left(C_{3}\right)=g^{-1}(\{\mathrm{R}, \mathrm{B}\}, G-F-S) \cup g^{-1}(\{\mathrm{R}, \mathrm{B}\}, F) ;
\end{aligned}
$$

Here, $C_{1}$ is a disjoint union of two cycles: $g^{-1}(\{\mathrm{R}, \underline{\mathrm{G}}\}, G-(S-F))$ and $(S-F) ; C_{2}$ a symetric difference of two cycles: $g^{-1}(\{\mathrm{R}, \underline{\mathrm{G}}\}, G-(S-F))$ and $F$; and $C_{3}$ is just $g^{-1}(\{\mathrm{R}, \mathrm{B}\}, G-(S-F))$, which is a cycle.

Let $E_{x}=g^{-1}(\{x\}, G-F-S)$, for $x \in\{\mathrm{R}, \mathrm{G}\}$. The length of the cover is $\mid G-F-$ $S|+2| E_{\mathrm{R}}|+| E_{\mathrm{G}}|+| F|+| g^{-1}(\{\mathrm{R}, \mathrm{B}\}, F)|+| S-F \mid$. Let $x \in\{\mathrm{R}, \underline{\mathrm{G}}\}$. If we restrict $G / F$ to the edges of $E_{x}$ we obtain a forest $T_{x}$. We can bound $\left|E_{x}\right|$ as $|V(G / F)|$ (which equals $\left.\sum_{i=1}^{\infty} d_{i}\right)$ minus the number of components of $T_{x}$. To bound the number of components of $T_{x}$ we just bound the number of isolated vertices of $T_{x}$ created by contracting a loop in $G$. Consider a vertex $v$ of $G / F$ created by contracting a loop. The two edges incident with $v$ have the same value under $f$. As the permutation of flow values was selected randomly, the probability that the two edges do not have value $x$ in $f$ is $2 / 3$. When creating the flow $g$ we only change flow values to $\mathrm{B}$, thus the two edges do not have value $x$ under $g$ with probability at least $2 / 3$. We have $\mathbb{E}\left(\left|E_{x}\right|\right) \leqslant\left(\sum_{i=1}^{\infty} d_{i}\right)-2 / 3 \cdot d_{1}=d_{1} / 3+\sum_{i=2}^{\infty} d_{i}$. Together with (1) the expected length of the cover is at most the expression in the claim 
statement and at least one of the six permutations leads to a cover with at most this length.

By combining Claims 5 and 6 we obtain the following.

Claim 7. Graph $G$ has a cycle cover of length at most

$$
\frac{16}{9} \cdot|G-S-F|+|S-F|+|F|+\frac{2}{9} \cdot d_{1}+\sum_{i=2}^{\infty} \frac{8}{15} \cdot i d_{i}
$$

Proof. We pick the cover from Claim 5 with probability $7 / 9$ and the cover from Claim 6 with probability $2 / 9$. The expected length of the cover is then bounded by the expression in the claim statement. For $i<20$ one has to check that the inequality holds separately for each coefficient. For $i \geqslant 20$ one can get the bound by ignoring the rounding. The bound is tight for $i=5$ and $i=20$. Thus note that if $F$ contains few components of length 5 or 20 a better bound can be obtained.

Now we are ready to prove Theorem 2 .

Proof of Theorem 2. If there is a loop incident with a vertex of degree more than 4, then we may remove it and cover it separately. Thus assume that each loop is incident with a vertex of degree 4 . Pick a proper spanning cycle $F$ of $G$ from the distribution from Lemma 3. Using $F$ we construct a cycle cover according to Claim 7 . As $F$ is a proper spanning cycle, $d_{1}=|F \cap S|$. Also $\sum_{i=1}^{\infty} i d_{i}=|F|$. Thus the length of the cover is at most

$$
\frac{16}{9} \cdot|G-S-F|+|S-F|+\frac{23}{15} \cdot|F|-\frac{14}{45} \cdot|F \cap S| .
$$

Due to the choice of $F$ we have $\mathbb{E}(|G-S-F|)=1 / 3 \cdot|G-S|, \mathbb{E}(|S-F|)=2 / 3 \cdot|S|$, $\mathbb{E}(|F|)=2 / 3 \cdot|G-S|+1 / 3 \cdot|S|$ and $\mathbb{E}(|F \cap S|)=1 / 3 \cdot|S|$. Thus the expected length of the cover matches the value in the theorem statement. This implies that there exists $F$ from the probability distribution from Lemma 3 such that the length of the cycle cover created using $F$ is bounded by the expression in the theorem statement.

\section{References}

[1] N. Alon, M. Tarsi. Covering multigraphs by simple circuits. SIAM J. Algebraic Discrete Methods, 6(3):345-350, 1985.

[2] J. C. Bermond, B. Jackson, F. Jaeger. Shortest coverings of graphs with cycles. J. Combin. Theory Ser. B, 35(3):297-308, 1983.

[3] B. Candráková, R. Lukotkka. Short Cycle Covers on Cubic Graphs by Choosing a 2-Factor. SIAM J. Discrete Math., 30(4):2086-2106, 2016.

[4] Z. Dvořák, D. Král, B. Mohar. Graphic TSP in Cubic Graphs. In 34th Symposium on Theoretical Aspects of Computer Science (STACS 2017), Schloss Dagstuhl-LeibnizZentrum für Informatik, 2017. 
[5] J. Edmonds. Maximum matching and a polyhedron with $(0,1)$ vertices. J. Res. Nat. Bur. Standards Sect B., 69B:125-130, 1965.

[6] G Fan: Integer 4-flows and cycle covers. Combinatorica, 37(1):1097-1112, 2017.

[7] H. Fleischner. Eulerian Graphs and Related Topics, Part 1, Elsevier, 1990.

[8] F. Jaeger. Flows and generalized coloring theorems in graphs. J. Combin. Theory Ser. B, 26(2):205-216, 1979.

[9] U. Jamshy, M. Tarsi. Short cycle covers and the cycle double cover conjecture. J. Combin. Theory Ser. B, 56(2):197-204, 1992.

[10] T. Kaiser, D. Král, B. Lidický, P. Nejedlý, R. Šámal. Short Cycle Covers of Cubic Graphs and Graphs with Minimum Degree Three. SIAM J. Discrete Math., 24(1):330-355, 2010.

[11] A. Raspaud. Flots et couvertures par des cycles dans les graphes et les matroïdes, PhD Thesis, Université Joseph-Fourier - Grenoble I, 1985. 